\title{
Paving the way to precision medicine for diabetic kidney disease: the PRIORITY trial
}

\author{
Meir Schechter ${ }^{1,2}$, Gil Leibowitz ${ }^{1,2}$, Ofri Mosenzon ${ }^{1,2}$ \\ ${ }^{1}$ Faculty of Medicine, Hebrew University of Jerusalem, Jerusalem, Israel; ${ }^{2}$ Diabetes Unit, Division of Internal Medicine, Hadassah Hebrew \\ University Hospital, Jerusalem, Israel \\ Correspondence to: Dr. Ofri Mosenzon. Diabetes Clinical Research Center, Hadassah University Hospital, PO Box 12000, Jerusalem, Israel 91120. \\ Email: ofrim@hadassah.org.il. \\ Comment on: Lancet Tofte N, Lindhardt M, Adamova K, et al. Early detection of diabetic kidney disease by urinary proteomics and subsequent \\ intervention with spironolactone to delay progression (PRIORITY): a prospective observational study and embedded randomised placebo-controlled \\ trial. Lancet Diabetes Endocrinol 2020;8:301-12.
}

Submitted Jun 11, 2020. Accepted for publication Jun 22, 2020.

doi: $10.21037 /$ atm-2020-117

View this article at: http://dx.doi.org/10.21037/atm-2020-117

\section{Introduction}

Diabetic kidney disease (DKD) is a common complication of both type 1 (T1D) and type 2 diabetes (T2D) (1). It is the leading cause of chronic kidney disease (CKD) worldwide and may result in end stage kidney disease requiring renal replacement therapy, either dialysis or kidney transplant. DKD is also associated with adverse cardiovascular (CV) outcomes and increased risk for mortality (2). However, many patients with diabetes do not develop advanced DKD even after prolonged follow-up and their response to different treatments may vary (3).

Angiotensin-converting enzyme inhibitors (ACEi) and angiotensin II receptor blockers (ARBs) are the mainstay therapy of $\mathrm{DKD}$, however their efficacy in preventing endstage renal disease is far from complete (4). More recently other treatments have been shown to protect the diabetic kidney, such as sodium-glucose cotransporter-2 inhibitors (SGLT2i) $(5,6)$, mineralocorticoid receptor antagonists (MRAs) (7-9), endothelin receptor antagonists (ERAs) (10) and glucagon-like peptide-1 receptor agonists (GLP-1 RAs) (11). Ideally, drugs should only be prescribed to patients who will benefit from treatment, and avoid unnecessary prescription to low-risk populations. This requires sensitive biomarkers that can accurately predict the onset of DKD and the subsequent response to treatment.

Increased urinary albumin to creatinine ratio (UACR), which results from hyperfiltration and glomerulopathy, is considered an early marker of DKD. Nonetheless, 40-70\% of patients may develop renal dysfunction without preceding albuminuria (12), and not all microalbuminuric patients progress to renal failure, suggesting the presence of large heterogeneity in the pathophysiology and course of DKD (13). The lack of sensitive and specific diagnostic tools for risk stratification limits personal tailoring of treatment, i.e., precision medicine.

CKD273 classifier is a urine proteomic quantitative marker based on a combination of 273 different peptides measured by capillary electrophoresis-mass spectrometry (CE-MS). It was developed and validated using urinary samples obtained from a large multinational cohort of 3,600 individuals. Analysis of urinary protein excretion using this platform effectively differentiated subjects with CKD of various etiologies from healthy controls, with a reported area under the curve (AUC) of 0.955 (14). Follow-up studies examined the predictive strength of CKD273 in different cross-sectional and longitudinal cohorts investigating renal outcomes. Importantly, increased CKD273 preceded the appearance of microalbuminuria by approximately 3.5 years. Urinary proteomic signature was also proposed to serve as a "liquid biopsy". Preliminary analyses showed that it can discriminate between various CKD etiologies and correlate with the severity of fibrosis detected in kidney biopsies. 
A more in depth review on CKD273 has been recently published (15).

The PRIORITY (Proteomic Prediction and Renin Angiotensin Aldosterone System Inhibition Prevention Of Early Diabetic nephRopathy In TYpe 2 Diabetic Patients With Normoalbuminuria; NCT02040441) trial investigated the efficacy of CKD273 in predicting the development of DKD in a prospective cohort. It also studied the effects of treatment with the MRA spironolactone on the development of microalbuminuria in high-risk patients according to CKD273.

\section{PRIORITY trial: design and main findings}

PRIORITY was a multinational prospective two part study: observational and interventional (16). Patients $(\mathrm{N}=1,775)$ with $\mathrm{T} 2 \mathrm{D}$, normoalbuminuria: UACR $<30 \mathrm{mg} / \mathrm{g}$ and estimated Glomerular Filtration Rate (eGFR) $>45 \mathrm{~mL} / \mathrm{min} / 1.73 \mathrm{~m}^{2}$ were stratified to high- and lowrenal risk according to a pre-specified single value of the CKD273 classifier. High-risk patients (CKD273 >0.154) were randomized to receive either low-dose spironolactone (25 mg; $\mathrm{n}=102)$ or placebo $(\mathrm{n}=107)$ in a double-blind manner. The primary endpoint was the development of confirmed microalbuminuria in the observational cohort (high $v s$. low risk). The main secondary objective of the trial was to assess spironolactone's effect on the rate of appearance of microalbuminuria (intervention cohort).

After a median follow-up of 2.5 (IQR, 2.0-3.0) years, patients classified as high-risk by CKD273 were more likely to achieve the primary outcome of confirmed microalbuminuria ( $H R=3.92$ ), even after adjusting for baseline risk markers (age, sex, HbA1c, systolic blood pressure, retinopathy, UACR and eGFR; HR =2.48). They also showed higher risk for some of the other pre-specified secondary renal parameters: occurrence of a single measurement of microalbuminuria, eGFR reduction of $>40 \%$, new CKD stage 3 or new CKD stage 4. Interestingly, high risk classification by CKD273 seemed to be associated with worse $\mathrm{CV}$ outcomes, albeit without reaching statistical significance. The authors also calculated the additive predictive accuracy of the CKD273 classifier for microalbuminuria. Receiver operator characteristic curve (ROC) for known baseline risk markers detailed above yielded an AUC of 0.76, which was increased to 0.78 by adding the CKD273. The difference was statistically significant $(\mathrm{P}=0.004)$, however the magnitude of the effect was modest.

Spironolactone failed to significantly improve the rate of new microalbuminuria in the intervention group. On the contrary, treatment was associated with increased risk for development of CKD stage 3, and significant hyperkalemia and gynecomastia.

\section{CKD273 classifier for risk stratification of DKD: advances and limitations}

PRIORITY is the first prospective multicenter trial verifying the predictive value of CKD273 for microalbuminuria in a cohort of patients with T2D and normoalbuminuria. Previous studies testing the accuracy of the assay and its use for prediction of DKD were all performed on retrospective cohorts, which might lead to biases in the interpretation of the data. The demonstration that CKD273 can reliably predict the development of DKD in a large prospective trial is a major achievement and an important step towards better stratification of DKD risk that will eventually enable making more precise clinical decisions as to treatment. There are several issues, however, that should be addressed before such an assay can be implemented in clinical practice.

The accuracy of a given biomarker depends greatly on the population tested, the selected cut-off, and the reliability of the test. All these factors should be integrated into a framework of pre- and post-test probability. In PRORITY, patients classified as high risk by CKD273 had almost four times increased risk to develop new microalbuminuria. Yet, the sensitivity of the test was still low, with the majority $(\sim 70 \%)$ of patients in the high-risk group not progressing to albuminuria within the time frame of the study. Of note, $9 \%$ and $8 \%$ of the low-risk patients developed microalbuminuria and impaired renal function (eGFR $<60 \mathrm{~mL} / \mathrm{min} / 1.73 \mathrm{~m}^{2}$ ), indicating that at this stage, the negative predictive value of the assay is still not sufficiently high to exclude future development of DKD. It is possible, nonetheless, that longer, register-based follow-up of PRIORITY population, as planned by the investigators, may result in better separation between the groups.

Adding CKD273 to baseline clinical characteristics enabled better prediction of future development of microalbuminuria, however the difference in the AUC of the ROC analysis was small, and the clinical significance of this small increment is questionable. The apparent small increase in DKD prediction with CKD273 compared to clinical assessment alone may be due to the unique design of the trial, which combined observational and interventional investigations within the same period. 
Consequently, patients and caregivers were not blinded to results of the CKD273 test; this may have led to more rigorous treatment of the patients that were classified as high-risk. Furthermore, compared to the low-risk group, more patients in the high-risk group were given ACEi/ ARBs (87\% vs. 61\%) at baseline by their treating physicians. Of note, it has been previously suggested that treatment with ACEi may increase CKD273 values (17). Previous studies provided some information on the validity and reproducibility of CKD273 (17), this however was not assessed in the clinical setup of PRIORITY. Further studies are required to address these issues.

At this stage, the availability of CKD273 is limited as it is performed in only three laboratories worldwide. Furthermore, the analysis is expensive (each test costs $\sim 850 €)$. Nevertheless, one can envision that further improvements of the accuracy of the assay, along with price reduction, will render the assay cost-effective, as early targeted treatment to high-risk patients may reduce future expenditures.

\section{Spironolactone for primary prevention of DKD}

In its second intervention part, PRIORITY investigated the effect of early spironolactone treatment to patients classified as high-risk by CKD273. The lack of statistically significant effect of spironolactone on the occurrence of new confirmed microalbuminuria can be attributed to the study being underpowered-identifying a smaller number of "high-risk", randomized patients (12.1\%) than initially expected (15\%) or following them for a too short duration.

Another explanation is the high dropout rate in the spironolactone group (25\%) compared with placebo (9\%) due to side-effects, such as hyperkalemia and gynecomastia. In addition, treatment with spironolactone was associated with occurrence of new CKD stage 3 (eGFR $<60 \mathrm{~mL} / \mathrm{min} / 1.73 \mathrm{~m}^{2} ; 32 \%$ vs. $14 \%$ ). This may result from the hemodynamic effects of Renin Angiotensin Aldosterone System (RAAS) pathway inhibition; nevertheless, this finding is worrisome, considering the fact that reduced renal function is associated with increased risk for mortality (2).

MRAs improve UACR in patients with T2D and albuminuria on top of ACEi/ARBs, but an effect on eGFR is lacking (8). Similar findings were observed with finerenone, a third generation non-steroidal and more selective MRA that is associated with lower risk of hyperkalemia and sex-hormone associated side effects (7). Finerenone's CV and renal efficacy is currently being investigated in large populations of patients with DKD (FIGARO-DKD and FIDELIO-DKD trials, respectively) (9). Further studies are required to assess whether early treatment with spironolactone and newer MRAs may prevent the development of microalbuminuria and/or advanced renal failure.

\section{Precision medicine in DKD: future perspective}

In recent years, several biomarkers have emerged as possible candidates to assist in the management of patients with T2D at risk for DKD. Such markers are based on differential expression of proteins, mRNAs and metabolites in blood or urine samples [reviewed in (18)]. In Table 1 we suggest a scheme that can be useful for systemic assessment of such biomarkers, and apply it to CKD273 in the context of PRIORITY.

Besides predicting outcome, such biomarkers may provide information as to disease course and pathophysiology. Many of the CKD273 peptides are associated with fibrosis, such as collagen fragments and extracellular matrix proteins. Interestingly, several studies indicated that renal protective therapies may normalize urinary proteomic signature, suggesting that fibrotic changes are reversible in early stages of DKD (15).

Sophisticated markers are sometimes used to predict the responses to various treatments. In DKD, however, such personal tailoring has been limited so far, due to both shortage in good treatment possibilities and suitable diagnostic tests. In recent years new therapies for DKD have emerged $(5-10,13,19)$. Remarkably, SGLT2i have been recently recommended by the American Diabetes Association (ADA) as the drug of choice for most patients with diabetes and renal risk (20). SGLT2i have been shown to consistently exert robust renal protective effects in different stages of DKD, including patients with normal UACR and eGFR $(5,6,19)$. It will be interesting to study whether CKD273 can predict the response to treatment in patients who are at risk to develop DKD, as well as in patients with established DKD.

PRIORITY is an important first step towards a new era of precision medicine in DKD, however further improvements in the predictive power of the assay are required before it can be clinically used to stratify the patient's risk and direct clinicians as to the choice of treatment. Integrating clinical parameters, along with proteomic-based analyses, such as CKD273 and probably additional large-scale genetic and metabolomic assays will eventually pave the way for precision medicine for DKD 
Table 1 A systemic assessment of diagnostic tools aid in achieving precise medical management: PRIORITY trial and CKD273 biomarker as prototypes

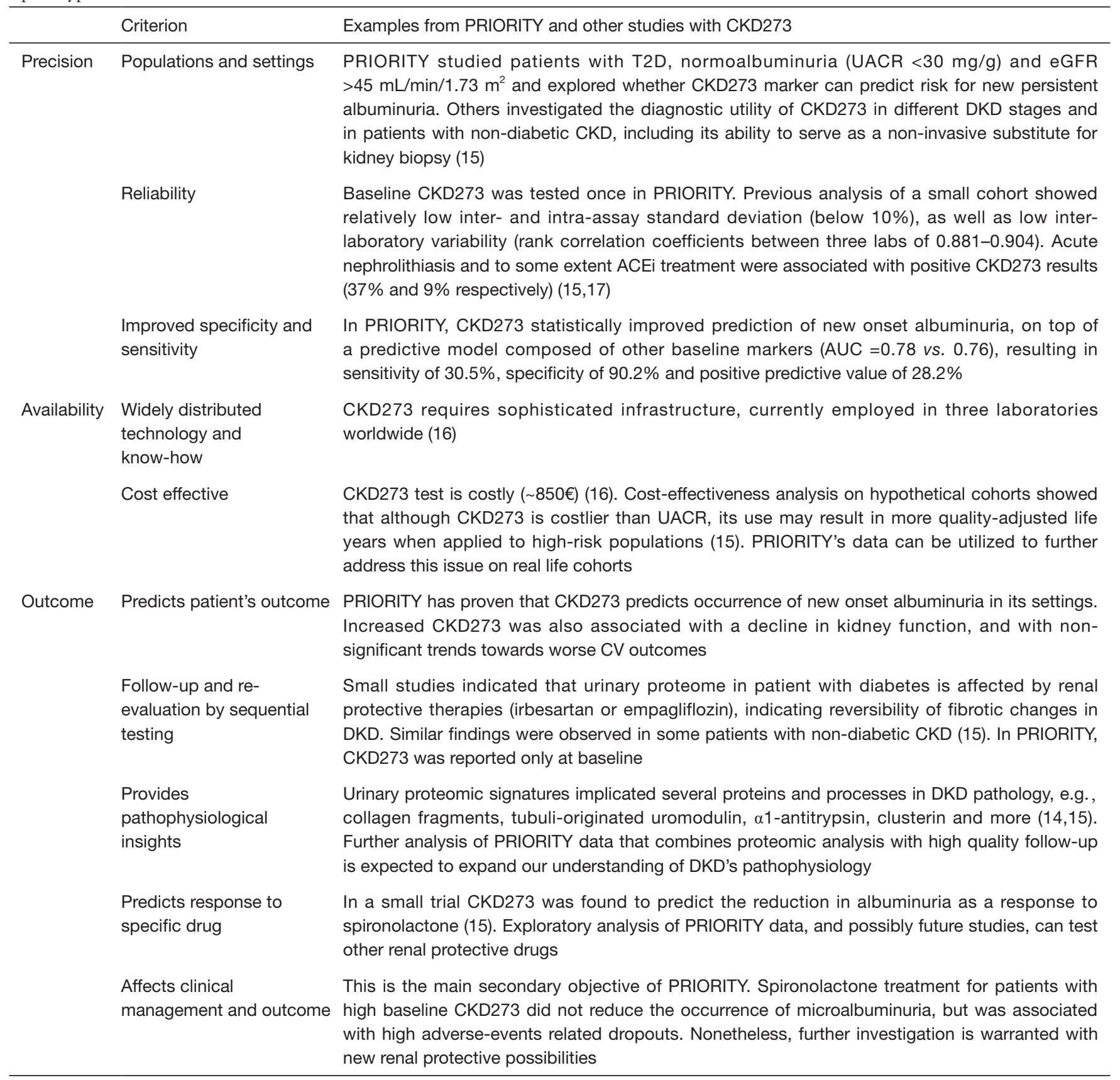

and probably also for non-DKDs.

\section{Acknowledgments}

The authors are grateful Ms. Rebecca Sprung for editing the manuscript and Prof. Erol Cerasi for providing critical review.

Funding: None.

\section{Footnote}

Provenance and Peer Review: This article was commissioned 
by the editorial office, Annals of Translational Medicine. The article did not undergo external peer review.

Conflicts of Interest: All authors have completed the ICMJE uniform disclosure form (available at http://dx.doi. org/10.21037/atm-2020-117). OM reports grants, personal fees and other from Novo Nordisk, grants, personal fees and other from AstraZeneca, personal fees and other from Eli Lilly, personal fees and other from Sanofi, personal fees and other from Merck Sharp \& Dohme, personal fees and other from Boehringer Ingelheim, other from BOL Pharma, outside the submitted work. The other authors have no conflicts of interest to declare.

Ethical Statement: The authors are accountable for all aspects of the work in ensuring that questions related to the accuracy or integrity of any part of the work are appropriately investigated and resolved.

Open Access Statement: This is an Open Access article distributed in accordance with the Creative Commons Attribution-NonCommercial-NoDerivs 4.0 International License (CC BY-NC-ND 4.0), which permits the noncommercial replication and distribution of the article with the strict proviso that no changes or edits are made and the original work is properly cited (including links to both the formal publication through the relevant DOI and the license). See: https://creativecommons.org/licenses/by-nc-nd/4.0/.

\section{References}

1. IDF Diabetes Atlas 9th edition 2019 [Internet]. [cited 2020 Apr 1]. Available online: https://www.diabetesatlas.org/en/

2. Chronic Kidney Disease Prognosis Consortium, Matsushita K, van der Velde M, et al. Association of estimated glomerular filtration rate and albuminuria with all-cause and cardiovascular mortality in general population cohorts: a collaborative meta-analysis. Lancet 2010;375:2073-81.

3. Afkarian M, Zelnick LR, Hall YN, et al. Clinical manifestations of kidney disease among US adults with diabetes, 1988-2014. JAMA 2016;316:602-10.

4. Lewis EJ, Hunsicker LG, Clarke WR, et al. Renoprotective effect of the angiotensin-receptor antagonist irbesartan in patients with nephropathy due to type 2 diabetes. $\mathrm{N}$ Engl J Med 2001;345:851-60.

5. Wanner C, Inzucchi SE, Lachin JM, et al. Empagliflozin and progression of kidney disease in type 2 diabetes. $\mathrm{N}$
Engl J Med 2016;375:323-34.

6. Mosenzon O, Wiviott SD, Cahn A, et al. Effects of dapagliflozin on development and progression of kidney disease in patients with type 2 diabetes: an analysis from the DECLARE-TIMI 58 randomised trial. Lancet Diabetes Endocrinol 2019;7:606-17.

7. Bakris GL, Agarwal R, Chan JC, et al. Effect of finerenone on albuminuria in patients with diabetic nephropathy: A randomized clinical trial. JAMA 2015;314:884-94.

8. Sun LJ, Sun YN, Shan JP, et al. Effects of mineralocorticoid receptor antagonists on the progression of diabetic nephropathy. J Diabetes Investig 2017;8:609-18.

9. Lytvyn Y, Godoy LC, Scholtes RA, et al. Mineralocorticoid antagonism and diabetic kidney disease. Curr Diab Rep 2019;19:4.

10. Heerspink HJL, Parving HH, Andress DL, et al. Atrasentan and renal events in patients with type 2 diabetes and chronic kidney disease (SONAR): a doubleblind, randomised, placebo-controlled trial. Lancet 2019;393:1937-47.

11. Kristensen SL, Rørth R, Jhund PS, et al. Cardiovascular, mortality, and kidney outcomes with GLP-1 receptor agonists in patients with type 2 diabetes: a systematic review and meta-analysis of cardiovascular outcome trials. Lancet Diabetes Endocrinol 2019;7:776-85.

12. Halimi JM. The emerging concept of chronic kidney disease without clinical proteinuria in diabetic patients. Diabetes Metab 2012;38:291-7.

13. Lin YC, Chang YH, Yang SY, et al. Update of pathophysiology and management of diabetic kidney disease. J Formos Med Assoc 2018;117:662-75.

14. Good DM, Zürbig P, Argilés A, et al. Naturally occurring human urinary peptides for use in diagnosis of chronic kidney disease. Mol Cell Proteomics 2010;9:2424-37.

15. Persson F, Rossing P. Urinary proteomics and precision medicine for chronic kidney disease: current status and future perspectives. Proteomics Clin Appl 2019;13:e1800176.

16. Tofte N, Lindhardt M, Adamova K, et al. Early detection of diabetic kidney disease by urinary proteomics and subsequent intervention with spironolactone to delay progression (PRIORITY): a prospective observational study and embedded randomised placebo-controlled trial. Lancet Diabetes Endocrinol 2020;8:301-12.

17. Mischak H, Vlahou A, Ioannidis JPA. Technical aspects and inter-laboratory variability in native peptide profiling: the CE-MS experience. Clin Biochem 2013;46:432-43. 
Page 6 of 6

18. Colhoun HM, Marcovecchio ML. Biomarkers of diabetic kidney disease. Diabetologia 2018;61:996-1011.

19. Perkovic V, Jardine MJ, Neal B, et al. Canagliflozin and renal outcomes in type 2 diabetes and nephropathy. $\mathrm{N}$ Engl J Med 2019;380:2295-306.

Cite this article as: Schechter M, Leibowitz G, Mosenzon O. Paving the way to precision medicine for diabetic kidney disease: the PRIORITY trial. Ann Transl Med 2020;8(24):1698. doi: 10.21037/atm-2020-117
Schechter et al. Precision medicine for DKD: PRIORITY trial

20. Buse JB, Wexler DJ, Tsapas A, et al. 2019 update to: management of hyperglycemia in type 2 diabetes, 2018. A consensus report by the american diabetes association (ADA) and the european association for the study of diabetes (EASD). Diabetes Care 2020;43:487-93. 